\title{
Systematic Review With Meta-analysis: Prompt Endoscopy As the Initial Management Strategy for Uninvestigated Dyspepsia in Asia
} (Aliment Pharmacol Ther 2015;41:239-252)

\section{Hye-Kyung Jung}

Department of Internal Medicine, Ewha Womans University School of Medicine, Seoul, Korea

\section{Summary}

Dyspepsia is a common gastrointestinal (GI) symptom with $10-20 \%$ of the population in the Asia-Pacific region. ${ }^{1}$ In Western countries, 40-60\% of individuals have a normal examination, whilst esophageal or gastric malignancies are detected in less than $1 \% .^{2}$ In Asia, the prevalence of Helicobacter pylori infection and upper GI malignancies are substantially high. ${ }^{3,4}$ However, among Asian patients with uninvestigated dyspepsia, the diagnostic value of alarm features and the appropriateness of prompt endoscopy are remained uncertainly. Chen et $\mathrm{al}^{5}$ performed a systematic review to evaluate the detection rate of organic diseases including upper GI malignancies and then to propose the proper initial diagnostic strategy in Asian dyspepsia patients. Among 2410 possible references, finally 18 papers $(n=152314)$ were included in the analysis of detection of organic disease by endoscopy among dyspeptic patients. This study revealed that overall malignancy detection rate was $1.3 \%$ (95\% confidence interval $0.8-2.1 \%$ ) and among cancer patients, $17.8 \%$ were younger than
45 years, $5.0 \%$ at age $<40$ years and $3.0 \%$ at age $<35$ years. The calculated detection rate of all organic diseases was $26.4 \%$ and that of peptic ulcer disease was $11.9 \%$ and esophageal disease was $5.5 \%$. However, alarm features were shown to yield moderate diagnostic accuracy with an area under curve of 0.74 . It meant limited accuracy for predicting malignancy. Therefore, authors concluded that an age of 35 years yielded relatively better diagnostic accuracy for malignancy.

\section{Comments}

Dyspepsia and its management remains a considerable socio-economic burden. Most Western guidelines recommended that alarm features at any age warranted prompt endoscopy and the cut-off age for recommendation of endoscopy in dyspeptic patients without alarm symptoms was 55 years as an initial strategy. In young patients, " $H$. pylori test and treat" or empirical proton pump inhibitors were recommended at first. ${ }^{6-8}$ However, " $H$. pylori test and treat" was unlikely to be beneficial in the high $H$. pylori positive area in aspect of cost-effectiveness. ${ }^{3}$ This large scale

Received: May 11, 2015 Revised: June 6, 2015 Accepted: June 23, 2015

(c) This is an Open Access article distributed under the terms of the Creative Commons Attribution Non-Commercial License (http://creativecommons. org/licenses/by-nc/4.0) which permits unrestricted non-commercial use, distribution, and reproduction in any medium, provided the original work is properly cited.

${ }^{*}$ Correspondence: Hye-Kyung Jung, MD, PhD

Department of Internal Medicine, Ewha Womans University School of Medicine, Mokdong Hospital, 1071, Anyangcheon-ro, Yangcheon-gu, Seoul 158-710, Korea

Tel: +82-2-2560-2632, Fax: +82-2-2655-2076, E-mail: junghk@ewha.ac.kr

Financial support: None.

Conflicts of interest: None.

ORCID: http://orcid.org/0000-0002-6653-5214 
meta-analysis was conducted on the basis of evidence-based medicine in the context of the insufficient existing data and authors clearly revealed important epidemiologic features. The malignancy detection rate in this study was significantly higher than that in Western countries (1.3\% vs $0.25 \%$ ). ${ }^{6}$ Based on these data, authors recommended the age cut-off for prompt endoscopy in dyspepsia patients as 35 year or less. Among dyspepsia patients who underwent the endoscopy, upper GI malignancy might be detected about $0.23 \%$ of patients less than 45 years and $0.03 \%$ of patients less than 35 years. However, establishing the appropriate age cut-off should be conducted taking into account the cost-effectiveness analysis with gastric cancer risk stratification. Moving from evidence to recommendations is another step to be needed a consensus.

In this systematic review, authors tried to validate the quality of reporting. They assessed the diagnostic accuracy by QUADAS criteria, ${ }^{9}$ however, most included studies in this analysis showed the "unclear" applicability, especially key factors, such as patient selection, reference standard and index test. ${ }^{9}$ And the results of meta-analysis in this study mainly depended on the 2 huge studies $\left(\mathrm{n}=120\right.$ 559). ${ }^{3,10}$ These 2 studies did not clearly define the definition of dyspepsia and conducted in single tertiary hospitals. Therefore, there might be some weakness, especially related with overestimating the benefits of the intervention being studied. ${ }^{11,12}$ Assessment of methodology quality is crucial and this is the essential part of as systematic review.

However, even though some limitations, this systematic review gives necessary and important information that is critical for decision making, especially to East Asian countries.

\section{References}

1. Ho KY, Kang JY, Seow A. Prevalence of gastrointestinal symptoms in a multiracial Asian population, with particular reference to re- flux-type symptoms. Am J Gastroenterol 1998;93:1816-1822.

2. Westbrook JI, Talley NJ. Diagnostic investigation rates and use of prescription and non-prescription medications amongst dyspeptics: a population-based study of 2300 Australians. Aliment Pharmacol Ther 2003;17:1171-1178.

3. Bai Y, Li ZS, Zou DW, et al. Alarm features and age for predicting upper gastrointestinal malignancy in Chinese patients with dyspepsia with high background prevalence of Helicobacter pylori infection and upper gastrointestinal malignancy: an endoscopic database review of 102,665 patients from 1996 to 2006. Gut 2010;59:722-728.

4. Eusebi LH, Zagari RM, Bazzoli F. Epidemiology of Helicobacter pylori infection. Helicobacter 2014;19(suppl 1):1-5.

5. Chen SL, Gwee KA, Lee JS, et al. Systematic review with meta-analysis: prompt endoscopy as the initial management strategy for uninvestigated dyspepsia in Asia. Aliment Pharmacol Ther 2015;41: 239-252.

6. Ford AC, Marwaha A, Lim A, Moayyedi P. What is the prevalence of clinically significant endoscopic findings in subjects with dyspepsia? Systematic review and meta-analysis. Clin Gastroenterol Hepatol 2010;8:830-837, e1-e2.

7. Talley NJ, Vakil N; Practice Parameters Committee of the American College of Gastroenterology. Guidelines for the management of dyspepsia. Am J Gastroenterol 2005;100:2324-2337.

8. Baron JH, Sonnenberg A. Hospital admissions and primary care attendances for nonulcer dyspepsia, reflux oesophagitis and peptic ulcer in Scotland 1981-2004. Eur J Gastroenterol Hepatol 2008;20: 180-186.

9. Whiting P, Rutjes AW, Reitsma JB, Bossuyt PM, Kleijnen. The development of QUADAS: a tool for the quality assessment of studies of diagnostic accuracy included in systematic reviews. BMC Med Res Methodol 2003;3:25.

10. Liou JM, Lin JT, Wang HP, et al. The optimal age threshold for screening upper endoscopy for uninvestigated dyspepsia in Taiwan, an area with a higher prevalence of gastric cancer in young adults. Gastrointest Endosc 2005;61:819-825.

11. Chalmers TC, Matta RJ, Smith H Jr, Kunzler AM. Evidence favoring the use of anticoagulants in the hospital phase of acute myocardial infarction. N Engl J Med 1977;297:1091-1096.

12. Schulz KF, Chalmers I, Hayes RJ, Altman DG. Empirical evidence of bias. Dimensions of methodological quality associated with estimates of treatment effects in controlled trials. JAMA 1995;273: 408-412. 